\title{
MORPHOLOGICAL OPERATION BASED DENSE HOUSES EXTRACTION FROM DSM
}

\author{
Y. Li ${ }^{\text {a, }}{ }^{*}$ L. Zhu ${ }^{\text {b }}$, K. Tachibana ${ }^{\text {b }}$, H. Shimamura ${ }^{\text {b }}$ \\ a Jiangsu Provincial Key Laboratory of Geographic Information Science and Technology International Institute for Earth System \\ Science, Nanjing University, 210000 Nanjing Jiangsu, China - liyan@ @ju.edu.cn \\ ${ }^{\mathrm{b}}$ PASCO corporation Japan, 1-1-2 Higashiyama, Megulo-ku Tokyo 153-0043, Japan - lin_zhu, hideki_shimamura@pasco.co.jp
}

Commission III, WG III/4

KEY WORDS: Mathematics, Transformation, Estimation, Detection, Building, Reconstruction, DSM

\begin{abstract}
:
This paper presents a method of reshaping and extraction of markers and masks of the dense houses from the DSM based on mathematical morphology (MM). Houses in a digital surface model (DSM) are almost joined together in high-density housing areas, and most segmentation methods cannot completely separate them. We propose to label the markers of the buildings firstly and segment them into masks by watershed then. To avoid detecting more than one marker for a house or no marker at all due to its higher neighbour, the DSM is morphologically reshaped. It is carried out by a MM operation using the certain disk shape SE of the similar size to the houses. The sizes of the houses need to be estimated before reshaping. A granulometry generated by opening-by-reconstruction to the NDSM is proposed to detect the scales of the off-terrain objects. It is a histogram of the global volume of the top hats of the convex objects in the continuous scales. The obvious step change in the profile means that there are many objects of similar sizes occur at this scale. In reshaping procedure, the slices of the object are derived by morphological filtering at the detected continuous scales and reconstructed in pile as the dome. The markers are detected on the basis of the domes.
\end{abstract}

\section{INTRODUCTION}

Buildings are basic and important geodesic features. Automatic geometric modeling using images or a Digital Surface Model (DSM) has been studied for many years, but it is still not sufficiently developed for practical applications. Semi-automatic building modeling has been widely applied when compared with entirely automated processes (Ameri et al., 2000; Förstner et al., 1997; Wang et al., 2011; Sampath et al., 2010). Mature 3D modeling is typically implemented using 2D models or 2D digital line graph data (DLG).

Our research focused on automated 2D geometric modeling. A number of algorithms have been proposed for building detection. However, the meaning of 'successfully detected' has not been investigated in detail, so a comparison between the relative performances of these algorithms is not available (Pfeifer et al., 2001). Existing building extraction techniques can be categorized into two main types. One type primarily extracts the buildings using image segmentation and pattern recognition on single or multiple images (Katartzis et al., 2008; Lari et al., 2007; Fazan et al., 2010; Hao et al., 2010; Lee et al., 2003). Many methods such as the watershed-plus-marker technique are not applicable to textured or very complex scenes. Additionally, they often lead to results that are not stable (Pesaresi et al., 2001). The other type uses the DSM or LIDAR point cloud data (Cheng et al., 2011; Awrangleb M. et al., 2012) to determine geometric characteristics such as size, height, and gradient. Pfeifer et al. (2001) summarized the methods that use laser or DSM to detect the buildings. Algorithms that extract houses often classify the data into a number of urban classes. One kind of method uses normalized DSM (nDSM) to extract off-terrain grids based on a height threshold, and then distinguishes buildings from trees using a classification method (Awrangleb M. et al., 2012; Hug et al., 1997; Elberink et al., 2000). Another kind of method first applies segmentation, and then uses a classification method to distinguish buildings from trees. The segmentation can be bottom-up (Matikainen et al., 2001) or region growing (Forlani et al., 2001), and is used to identify segments above the terrain. Both of these methods use the height signal as a principal identifier.

Mathematical morphology (MM) has been demonstrated to be an efficient tool for DSM data. Recently published papers have recognized the effectiveness of mathematical morphology when extracting objects of given shapes (Valero et al., 2010; Sagar et al., 2000; Soille et al., 2007; Sathymoorthy et al., 2007). More studies have investigated building extraction over the last two decades (Rottensteiner et al., 2002; Brunn et al., 1997; Zhan etal., 2002; Sportouche et al., 2011). Zhan et al. (2002) proposed a volumetric approach, where the DSM is considered in multiple layers, i.e., slices. The connected component in each slice is labeled. Such a component is classified as belonging to a building if the components in the vertically neighbored slices have similar sizes and centers of gravity. Rottensteiner et al. (2002) extracted initial building masks using a binary segmentation based on a height threshold. The masks still contained vegetation and other objects, so they used the binary morphological opening to separate and filter out small thin objects. They then analyzed the DSM textures to eliminate vegetation and other areas. The final individual building regions were determined using a connected component analysis. A decreasing-sized structural element for morphological opening helped to split the regions that corresponded to more than one roof plane. Brunn et al. (1997) used a binary classification to distinguish vegetation from buildings, by applying morphology to derive closed areas on the surface normal variance and select valid vegetation segments. $\mathrm{Li}$ et al (2014) used a modified white top-hat transform with directional edge constraints to filter airborne LiDAR data and extract the ground points. They proposed a novel algorithm that used the modified white top-hat (MWTH) transform with directional edge constraints to automatically extract the ground points from airborne LiDAR data. Other studies used MM on a multi-spectral image instead of DSM, and produced good results. Lefevre (2007) used very 
high-resolution (VHR) gray level images as the input data to extract the buildings. They transformed the image to a binary image where the bright or dark buildings can be separated from the others. Then a morphological filter was applied to remove the objects that are smaller than a given minimal size of a building. Huang (2011) proposed a morphological building index (MBI) to represent the brightness, size, and orientation of the buildings using the reconstruction, differential morphological profile (DMP), and directionality. However, the MBI feature image is segmented according to a threshold to extract the buildings, which is not flexible or adaptive. Besides, DMP cannot reflect the height characteristics, only the width or size of the building. Meng (2012) proposed a method for detecting the residential land use of buildings from LiDAR data, aerial photography, and road maps. They used a morphology-based building detection algorithm to identify buildings from LiDAR and aerial photographs. However, this was not sufficient and a supervised C4.5 decision tree analysis was implemented to finally separate the residential buildings. To summarize, the above algorithms and methods generally consider LiDAR data, which are more accurate than photographic-derived DSM data in terms of the original direction and height. However, more than one method is often required to separate buildings that are close together. These techniques may not solve the problem when applied to the DSM of dense residential areas, where an abundance of joined-together houses may affect the accuracy of the DSM. However, a DSM can be simply and economically derived from photography and can cover a large area, so it is a practical choice for building extraction and modeling.

\section{METHODS}

As we know, the metropolises are characterized by various building types and complex spatial distributions. There are always tens of thousands of buildings in some districts of such cities, thus manually modeling them is labor intensive. Besides of the computational workload, the densely housing areas are difficult to handle in the automatic modelling. Houses in a digital surface model (DSM) are almost joined together in such areas, and most segmentation methods cannot completely separate them.

Taking Tokyo as the case, this paper introduces an MM-based building extraction system for complex dense housing areas. Sapporo locates in is the northern Japanese island of Hokkaido, at $43^{\circ} 4^{\prime} \mathrm{N}$ and $141^{\circ} 21^{\prime} \mathrm{E}$. The area of Sapporo is $1121 \mathrm{~km}^{2}$, including 10 districts. It is the administrative center of the Hokkaido, and also the fourth-largest city in Japan by population. It has the appearance of a modern city. The city spread along the river which divides it into northern and southern parts. There are two main streets intersecting in the city centre. There geographic features divide the city into several natural and different regions with various building types. There are many low-rise high-density housing areas in the suburbs of the city. It is more challenging to model these houses compared with low-density housing. The extraction is complex because of the surrounding trees, objects, and nearby houses. The house may be so close that they appear attached in less accurate DSMs. As a result, it is difficult to isolate one house from the others. We have addressed it in both our scale analysis and extraction algorithm.

\subsection{Overview of the technique}

Here is the general flowchart of the technique in Figure 1.

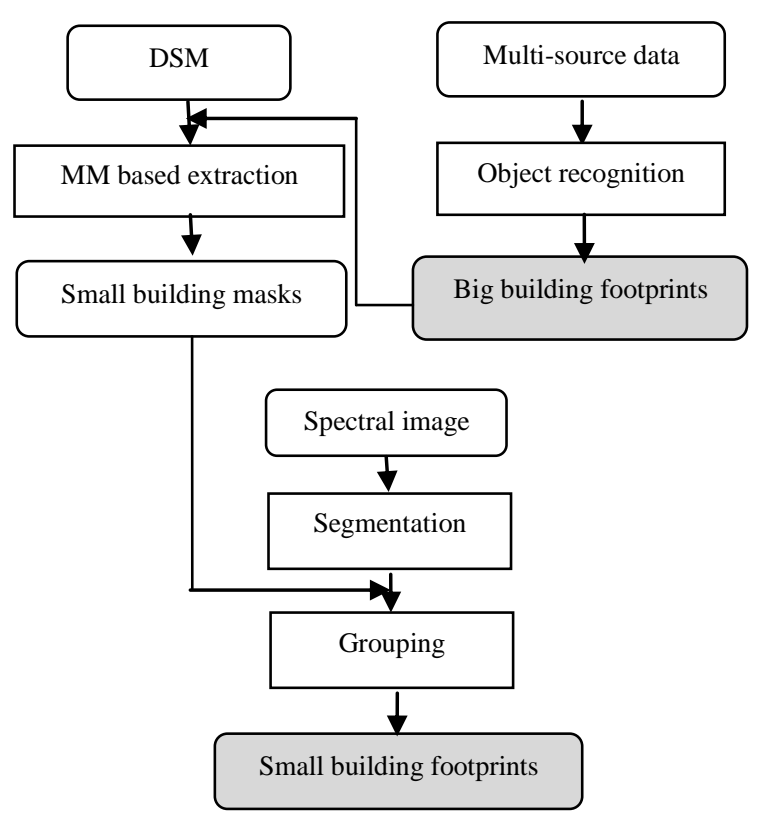

Figure 1 The general technique illustration

It respectively generates the small building footprints and the big building footprints through two algorithm branches. The reason to take this stratacy is that there will be big or high residential buildings as well as the small ones in one residential area, while the distributions of them are different. The big buildings are always locate sparsely comparing with the small ones. It is better to extract the big buildings firstly by easy processing, to avoid their disturbing to the complicated extraction of the small houses. Thus firstly, the multiple data including the RGB image and DSM are integrally segmented into the relatively big objects, which are more robust and reliable than pixels in spectral characteristics and furthermore, can provide contour information. The objects of big buildings will be characterized by big areas and straight contours. Criteria of such features are set up to recognize those objects and big building footprints are generated by them.

Then, the MM based small house mask extraction is carried out on DSM. They will be used to group the fragmented objects derived from the image segmentation into house footprints. DSM can be taken as a surface in the 3D space when the height corresponds to the z-axis, and also a gray level image when the height corresponds to the brightness. The buildings and other off-terrain objects show as the convex objects in the surface or the bright objects in the gray level image. It is relatively easy to extract the intact buildings from the DSM comparing with from the spectral image. However, the shapes of the buildings derived from the DSM are less perfect than from the image. That is why we extract building mask firstly instead of extracting the buildings directly from the image. The MM based mask extraction can be described clearly by Figure 2 . 


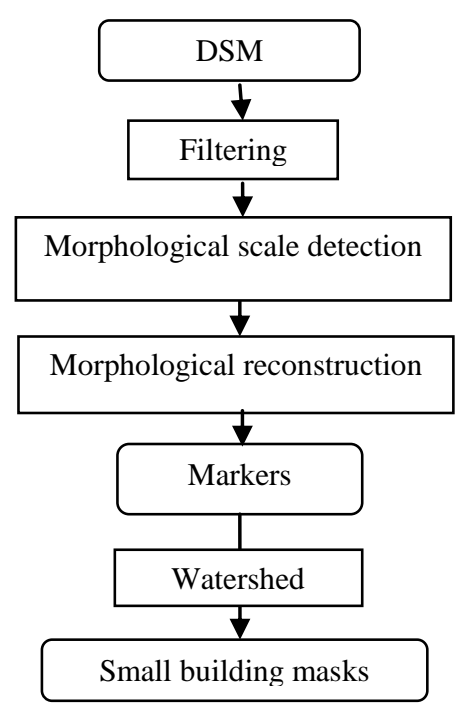

Figure 2 Flowchart of MM based mask extraction

The smoothed DSM has been morphologically filtered to derive DTM and NDSM. A structure element (SE) of 100-by-100 was used to do opening to the smoothed DSM to get the DTM, and a SE of 60-by-60 was used to smooth the DTM. NDSM is derived by subtracting DTM from DSM. The pixels of the NDSM with values under zero are taken as the ground pixels. Others are taken as the off-terrain pixels.

Because the small houses within a neighbourhood generally have similar sizes, particularly in the modern city block with good planning, there will be some key scales corresponding to them. A granulommetry technique is used for scale detection for NDSM, which will be described in the next part. It is a kind of MM method. However, even if the key scales are acquired, the joint effect of the dense houses in NDSM still remains as the problem for correct separation of the houses. For this, an MM reshaping processing for the houses is implemented to reconstruct the domes of the houses. Markers and masks can be detected and segmented on the basis of the domes.

In the procedure of small house mask extraction, a big building map was used to filter the NDSM so that the values of the big building pixels can be set to 0 . The small house mask extraction then has completely nothing to do with the big buildings.

\subsection{Granulometry based scales detection}

The gray level (GL) image/DSM can be considered as a collection of level images, each corresponding to a horizontal profile of the GL image/DSM surface. The inner pixels have a value of 1 , the outer pixels are 0 , and the GL image/DSM is a sum of all the level images. Morphological operations can be applied to each level image, and output a sum of the results. Vincent and Pierre stated that an opening removes some peaks and crest lines, whereas a closing tends to fill in basins and valleys (Vincent et al., 1991). Morphological reconstruction is particularly a powerful tool, which can be used to isolate certain features within an image, based on the manipulation of a mask image $f$ and a marker image $g$ (Sathymoorthy et al., 2007).

The available data include visible light airborne images of Sapporo, Japan with a $0.2-\mathrm{m}$ resolution, and the DSM derived through photogrammetry with a $0.5-\mathrm{m}$ resolution (which is registered to the images). The spatial or height accuracy of the DSM is not as good as the original images. The obvious advantage is that the signal reflects the height directly, and is immune to color variations caused by the shade of a certain object. Thus, we used the DSM to detect the markers that correspond to the regions of the houses and generate the building masks. We used the multiple-spectral image to accurately acquire the house outlines with the help of the masks.

The scales of the houses can be detected using granulometry analysis. A granulometry is a kind of morphological histogram (Serra 1982). It is computed using a sequence $\left(\Gamma_{S, n}\right)$ of morphological openings with a structuring element $S$ of increasing size $n$. It reflects the main sizes of the objects in the scene using obvious increases in the curve. Unlike Santibañez (2012) and Mura (2011), we define a granulometry as the mapping of a sequence ( $\left.\Gamma_{S, n}\right)$ of morphological opening-by-reconstruction with structuring element $S$ of increasing size $k$. That is,

$$
\Gamma_{S, n}=\left(v\left(\rho_{I}\left(\varepsilon_{k S}(I)\right)\right)\right)_{k \in[1, n]}
$$

where $k S$ refers to the $S$ dilated $k$-times, and v(o) refers to the surface function (which returns the total height of the non-zero pixels and the volume of o). As shown in Figure 3 (a), the value of $\Gamma_{S, n}$ increases as the size of the structure element increases. The difference between the original image volume and $\Gamma_{S, n}$ exactly corresponds to the volume of the cut-off domes of the objects under that size. The volume less than a certain size may contain noise and small objects such as trees or cars. There may be no buildings under the smaller sizes. When the size matches a building, there is an obvious steep increase in the granulometry. The difference between $\Gamma_{S, n}$ and $\Gamma_{S, n-1}$ is called the pattern spectrum (PS), and is also known as a differential morphological profile (Lefèvre et al., 2007). It represents the reduced volume between the two scales. The peak of the PS curve reflects the main scale of the objects in the image. We propose to construct the granulometry using opening-by-reconstruction instead of opening to implement the scale mining. The difference between opening-by-reconstruction and opening is that a bright object in the opened-by-reconstruction image will remain the same size at each scale, while in the opened image it becomes smaller and more compact. In other words, the histogram of the opening-by-reconstruction operation reflects the real volume change caused by removed domes.

Figure 3(a) gives an example of a typical granulometry profile of a DSM. The $x$-axis corresponds to the radius of the structural element (SE), and the y-axis corresponds to the granulometry number. Figure 3(b) shows the PS of Figure 3(a). There is more than one peak in the PS curve, because there are buildings at multiple scales. The SE is a disk with a radius of one pixel. The highest peak occurs at 23 , which indicates the main scale of most houses. The big increases within a range of continuous scales in the granulometry curve reflect the large amount of dome removal in the gradual morphological process. We expect that we can extract all the houses using this scale range. Thus, we propose a scale range detection method that uses the PS curve, described as follows. The start of the range $\left(s_{0}\right)$ is assigned as the point where there is a break in the increases of the granulometry curve. The cumulative average of the PS is defined as 


$$
m_{c}(n)=\frac{1}{n-1} \sum_{j=2}^{n} \Gamma_{S, j}-\Gamma_{S, j-1}, n=2,3, \ldots
$$

Obviously $m_{c}(n)$ is the slope of the line connecting the head of the granulometry curve to the point corresponding to $n$. When the local slope of the curve at point $n$ is obviously higher than $m_{c}(n), n$ is detected as $s_{0}$. The peak scale can be taken as the end of the scale range, $s p$.

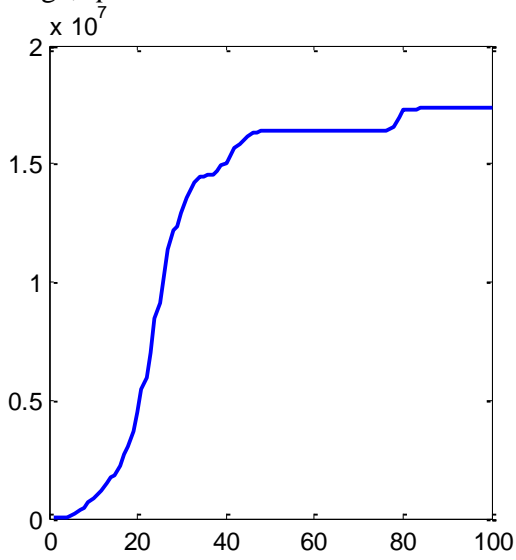

(a) Granulometry

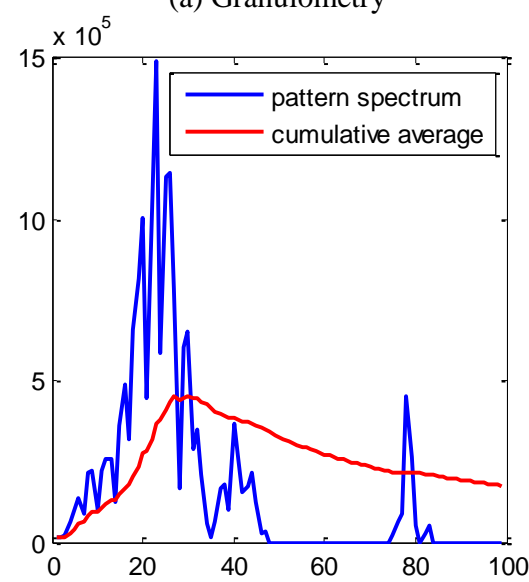

(b) PS

Figure 3 Granulometry and PS using opening-by-reconstruction

\subsection{Dome reshaping}

The reason of dome reshaping is presented in the beginning of part 2. As we know, morphological operations change the height and shape of an object in the level images. For instance, an SE disk shape makes the corners of a square object disappear by opening. Opening can shrink and round general objects.

Therefore, we filtered the DSM image to change the building shapes and reconstruct the building domes to easily detect the markers. To this end, we propose an iterative differential opening-based morphological filtering algorithm. The continuous opening to the DSM shrinks the shape of an off-terrain object and makes it more round. This separates connected houses. Then, we partly filter the object slice at a certain scale by subtracting the opened DSM from the last iterative result. The sizes of the SE used in this stage are identical to the scales detected in Section 2.2. Some thin edges remain after differencing, which correspond to the outside of the non-rounded objects. We use opening to erase this noise from the filtered objects. The reshaped domes will look like the shapes in Figure 4 (b), while Figure 4(a) is the NDSM.
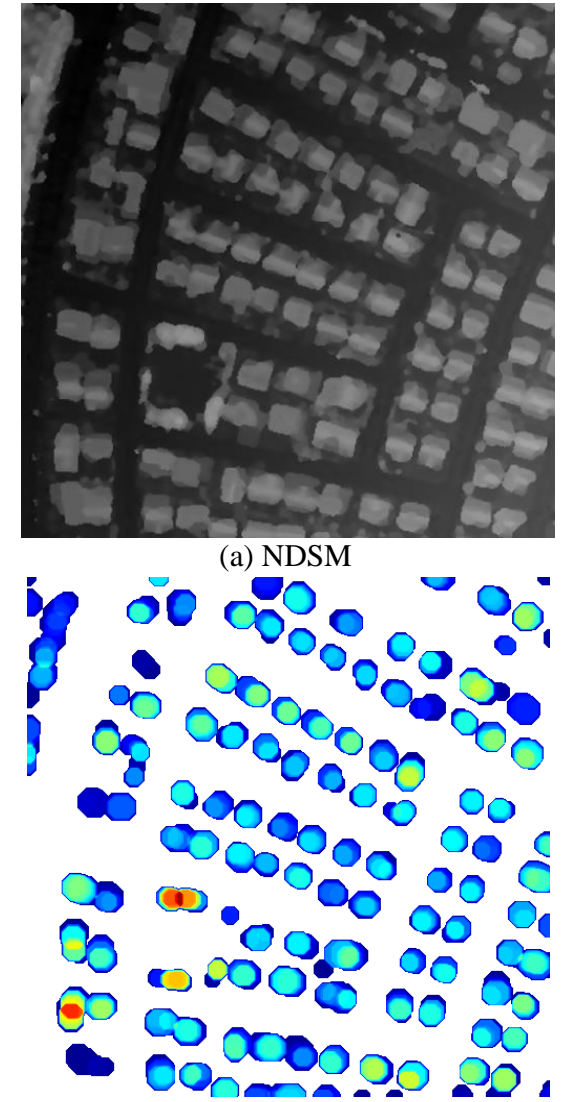

(b) Reconstructed domes

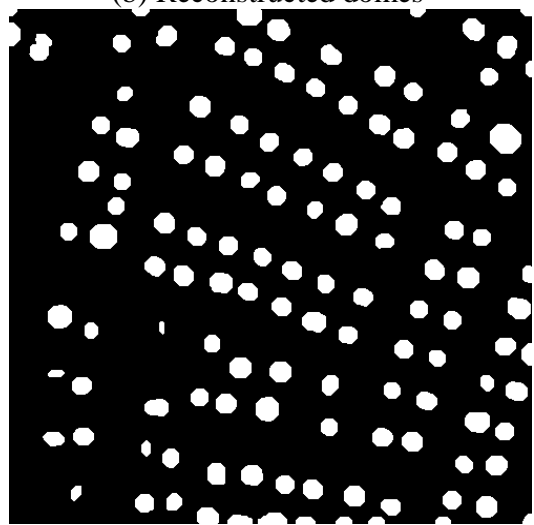

(c) Markers

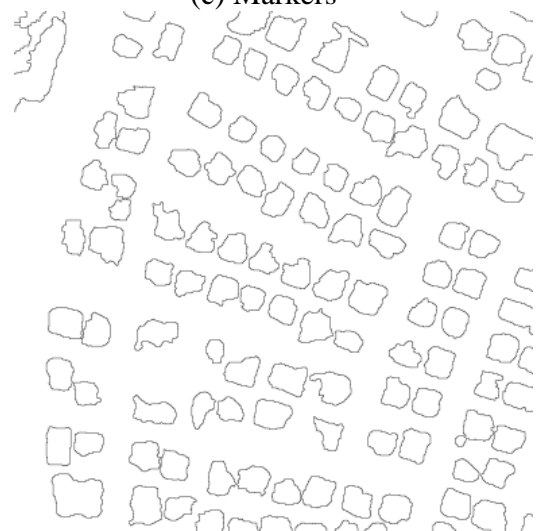

(d) Masks 


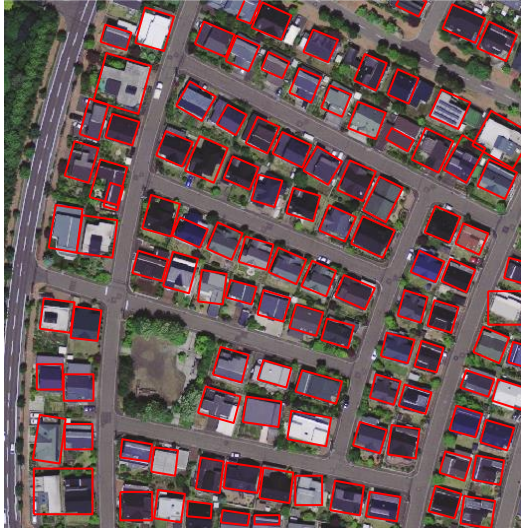

(e) Models

Figure 4 The morphological computation results: (a) reconstructed houses, (b) markers, and (c) masks.

\subsection{Mask extraction}

The markers present as a round spot that indicates the basic location of a building, as shown in Figure 4 (c). A mask is a region that covers the basic shape of a building. We used watershed segmentation on the gradient of the DSM (GDSM) to segment the building masks, by setting the markers of the local minima of the GDSM (i.e., the catchment basins). The classic immersion algorithm proposed by Vincent and Soille (1991) can give the desired result. The mask map for the test data in Figure 3 is shown in Figure 4(d).

In our case, the near infrared image is not available, so we developed a normalized difference index to classify vegetation and artificial surfaces using R, G, and B channels.

\section{RESULTS}

We investigated the effectiveness of the proposed method using some experimental results on Japanese test datasets, which contained various types of house distributions. Figure 4 shows the typical result of the data sets. Figure 4(e) draws the polygon models of the buildings in this area in red on the RGB image. The polygon modelling method is not the goal of this paper and is omitted. The result shows that all the houses were correctly labelled and separated. The regular models fit the houses very well, in the sense of both the orientations and the sizes. Figure 5(a) shows the clip of the NDSM of another place in Sapporo, where big residential buildings and small dense houses are both present. Figure 5(b) displays the polygon models in red on the RGB image. Only two houses are mixed as one, while other houses and buildings are extracted.

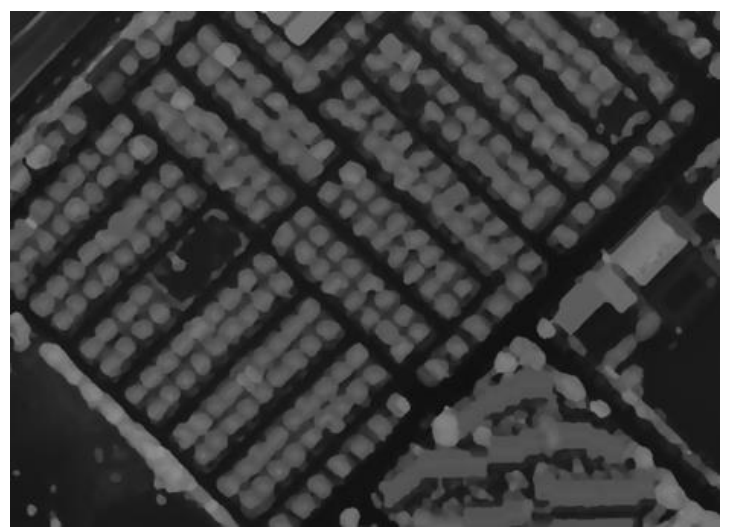

(a) NDSM

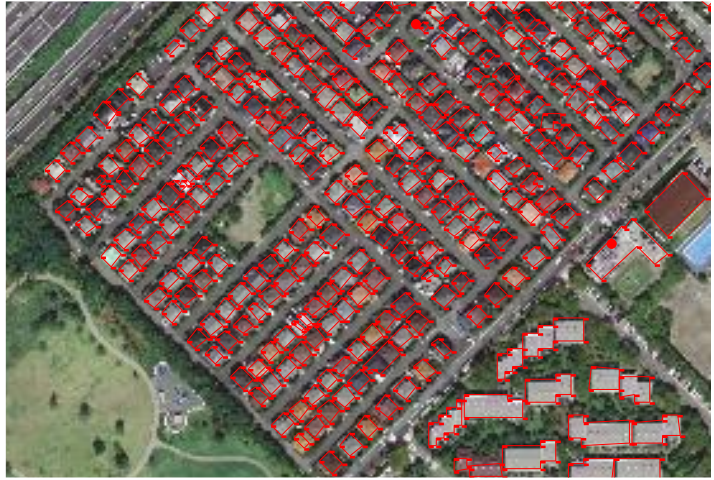

(b) Models

Figure 5 The models of the extracted buildings

\section{CONCLUSIONS}

The objective of this work was to extract houses from DSM of high-density residential areas and avoid combining them into a single object. The process is difficult, because houses may be attached in the DSM. They are concave in the DSM surface but higher than the true ground. It is quite difficult to identify these as isolated houses. We have proposed a new method. A dome is reconstructed for an off-terrain object using a collection of slices that are derived using iterative differential openings of the DSM. The sizes of the structural elements in the morphological implementation are determined by the scale range of the houses in the residential area. We proposed a modified granulometry analysis to detect these scales. Our experiments on real data demonstrate that the scale detection method is robust to different data, because it yields a scale range instead of a single value. This scale range guarantees that the slices can be extracted for some house at least once, and thus be labeled as the marker. Joins between houses correspond to a peak in the pattern spectrum or a sharp change of the granulometry. Thus, they are not generally extracted as slices. As a result, attached houses can be separated.

\section{REFERENCES}

Ameri B., and Fritsch D., 2000, Automatic 3d building reconstruction using plane-roof structures, Proc. of ASPRS annual conference, 22-25 May 2000, Washington DC, pp.22-26.

Awrangleb M., Zhang C., Fraser C.S., 2012, Building detection in complex scenes through effective separation of buildings from trees, Photogrammetric Engineering \& Remote Sensing,78(7):729-745.

Brunn A., and Weidner U., 1997, Building extraction from Digital Surface Models, IAPRS 3D Reconstruction and Modeling of Topographic Objects, Stuttgart Germany, 17-19 September 1997, Vol. 32, Part 3-4W2.

Cheng L., J. Gong, M. Li, Y. Liu, 2011, 3D building model reconstruction from multi-view aerial imagery and LiDAR data, Photogrammetric Engineering \& Remote Sensing, 77(2):125-139.

Elberink S. O. and Maas H.G., 2000, The use of height texture measures for the segmentation of airborne laser scanner data, International Archives of Photogrammetry and RS, 33/3A, Amsterdam, The Netherlands. 
Fazan A. J. and Poz A. P. D., 2010, Building roof contours extraction from aerial imagery based on snakes, and dynamic programming, FIG Congress 2010 Facing the Challenges Building the Capacity, 11-16 April 2010, Sydney, Australia, pp. 11-16,.

Forlani G.and Nardinocchi C., 2001, Building detection and roof extraction in laser scanning data, International Archives of Photogrammetry and RS, 34 .

Förstner W., and Plüumer L. (eds) 1997, Semantic Modeling for the Acquisition of Topographic Information from Images and Maps. Birkhüauser Verlag, Bonn, Germany.

Hao T., Yang J., and Wang Y., 2010, Towards automatic building extraction: Variational level set model using prior shape knowledge, Acta Automatica Sinica, 36(11):.1502-1511.

Huang X., Zhang L., 2011, A multidirectional and multiscale morphological index for automatic building extraction from multispectral Geoeye-1 imagery, Photogrammetric Engineering \& Remote Sensing, 77(7):721-732.

Hug C. and Wehr A., 1997, Detecting and identifying topographic objects in imaging laser altimetry data, International Archives of Photogrammetry and RS, 32/3-4W2.

Katartzis A., and Sahli H., 2008, A Stochastic Framework for the Identification of Building Rooftops Using a Single Remote Sensing Image, Geoscience and Remote Sensing, IEEE Transactions on, $46: 259-271$.

Lari Z., and Ebadi H., 2007, Automated building extraction from high-resolution satellite imagery using spectral and structural information based on artificial neural networks, ISPRS Hannover Workshop 2007 High-Resolution Earth Imaging for Geospatial Information, 29 May-1 June 2007, Hannover, Germany.

Lee D.S., Shan J., and Bethel J.S., 2003, Class-guided building extraction from Ikonos imagery, Photogrammetric Engineering and Remote Sensing, 69(2): 143-150.

Lefèvre S., Weber J., and Sheeren D., 2007, Automatic building extraction in VHR images using advanced morphological operators, Proc. IEEE/ISPRS Joint Workshop Remote Sens. URBAN, 11-13 April 2007, Paris France, pp. 1-5.

Li Y., Yong B., Wu H., An R., Xu H., Xu J., He Q., 2014, Filtering airborne LiDAR data by modified white top-hat transform with directional edge constraints, Photogrammetric Engineering \& Remote Sensing, 80(2): 133-141.

Matikainen L., Hyypp“a J., and Hyypp“a H., 2001, Automatic detection of buildings from laser scanner data for map updating, International Archives of Photogrammetry and RS, 34/3-W13.

Meng X., Currit N., Wang L., Yang X., 2012, Detect residential buildings from LiDAR and aerial photographs through object-oriented classification, Photogrammetric Engineering \& Remote Sensing, 78(1): 35-44.

Mura M. D, Benediktsson J. A, Chanussot J., and Bruzzone L., 2011, The evolution of the morphological profile: from panchromatic to hyperspectral images, Prasad S., Bruce L. M., and Chanussot J. Editors, Optical Remote Sensing, Springer, Volum3, pp.123-146.
Pesaresi M., Benediktsson J.A., 2001, A New Approach for the Morphological Segmentation of High-Resolution Satellite Imagery, IEEE Transactions on Geoscience and Remote Sensing, 39 (2):309-320.

Pfeifer N., Rutzinger M., Rottensteiner F., Muecke W., and Hollaus M., 2007, Extraction of building footprints from airborne laser scanning: Comparison and validation techniques, Urban Remote Sensing Joint Event, 2007, 11-13 April, Paris, France, pp.1-9.

Rottensteiner F., Briese C., 2002, A new method for building extraction in urban areas from high-resolution LiDAR data, International Archives of the Photogrammetry, Remote Sensing and Spatial Information Sciences, Vol. XXXIV,pp. 295-301.

Sagar, B.S.D., Venu, M., and Srinivas, D., 2000, Morphological operators to extract channel networks from digital elevation models, International J. RS, 21(1): 21-29.

Sampath A and Shan J., 2010, Segmentation and Reconstruction of Polyhedral Building Roofs From Aerial LiDAR Point Clouds, IEEE TRANSACTIONS ON GEOSCIENCE AND REMOTE SENSING, 48(3):1554-1567.

Santibañez J. D. M., Duarte M. G, Meléndez D. J. G. and Sánchez A. R. J, 2012, Chapter 9,Comparison of Granulometric Studies of Brain Slices from Normal and Dissociated Strabismus Subjects Through Morphological Transformations, Advances in Brain Imaging, edited by Vikas Chaudhary, ISBN 978-953-307-955-4, Published: February 1, 2012.

Sathymoorthy D., Palanikumar R., and Sagar B. S. D., 2007, Morphological segmentation of physiographic features from DEM, International Journal of Remote Sensing, 28(15): 33793394.

Serra J., Image Analysis and Mathematical Morphology. Academic Press, 1982.

Soille P. and Grazzini J., 2007, Extraction of River Networks from Satellite Images by Combining Mathematical Morphology and Hydrology, CAIP'07 Proceedings of the 12th internati-nal conference on Computer Analysis of Images and Patterns, 27-29 August, Vienna Austria, pp 636-644.

Sportouche H., Tupin F., Denise L., 2011, Extraction and Three-Dimensional Reconstruction of Isolated Buildings in Urban Scenes From High-Resolution Optical and SAR Spaceborne Images, Geoscience and Remote Sensing, IEEE Transactions on, 49(10): 3932- 3946S.

Valero S., Chanussot J., Benediktsson J.A., Talbot H., Waske B., 2010, Advanced directional mathematical morphology for the detection of the road network in very high resolution remote sensing images, Pattern Recognition Letters, 31(10): 1120-1127.

Vincent L., Soille P., 1991, Watersheds in digital spaces: An efficient algorithm based on immersion simulations, IEEE Trans on Pattern Analysis and Machine Intelligence, 13(6):583-598.

Vincent L..1993, Morphological Grayscale Reconstruction in Image Analysis: Applications and Efficient Algorithms, IEEE Transactions on Image Processing, 2 (2): 176-201.

Wang Q., Jiang Z., Yang J., Zhao D., and Shi Z., 2011, A Hierarchical Connection Graph Algorithm for Gable-Roof 
The International Archives of the Photogrammetry, Remote Sensing and Spatial Information Sciences, Volume XL-3, 2014 ISPRS Technical Commission III Symposium, 5 - 7 September 2014, Zurich, Switzerland

Detection in Aerial Image, IEEE Geoscience and Remote Sensing Letters, 8(1):177-181.

Zhan Q., Molenaar M., and Tempfli K., 2002, Building extraction from laser data by reasoning on image segements in elevation slices, International Archives of Photogrammetry and RS, 35 . 